\title{
HYDRODYNAMIC STATE OF ART REVIEW: ROTOR - STATOR MARINE PROPULSOR SYSTEMS DESIGN
}

\author{
Przemysław Król \\ Ship Design and Research Centre, Gdańsk, Poland
}

\begin{abstract}
The paper covers the important topic of rotor-stator propulsor system design and operation. For the stand-alone marine screw propeller, both the design criteria for loading distribution and the theoretical efficiency limits are well described in the basic literature. This is in contrast to the combined propulsor system like a propeller cooperating with a pre-swirl device. The paper describes the current state of the art, summarising results obtained by various researchers by installing energy-saving devices on particular vessels. The design methods utilised are briefly outlined, with the main characteristics underlined. Rough analysis of the gathered data confirms the expected trend that a higher efficiency gain due to ESD installation is possible for a higher propeller loading.
\end{abstract}

Keywords: : energy-saving device, guide vane, pre-swirl stator, propulsor design, efficiency

\section{INTRODUCTION}

A vast amount of literature exists that covers the topic of marine propulsor design and its optimization. Due to rising environmental requirements, more attention is being paid nowadays to the propulsor efficiency. Consequently, energy-saving devices (ESDs) are gaining increasing interest from researchers and marine engineers. Important types of these are pre- and post-swirl devices, intended to recover rotational energy losses occurring during screw propeller operation. This paper addresses ESDs of various forms, but the main focus is on pre-swirl stators (PSS). The most important types of ESDs are presented in Fig. 1.

The pre-swirl stator is a system of rigid lifting foils, located before the operating propeller. These are meant to produce

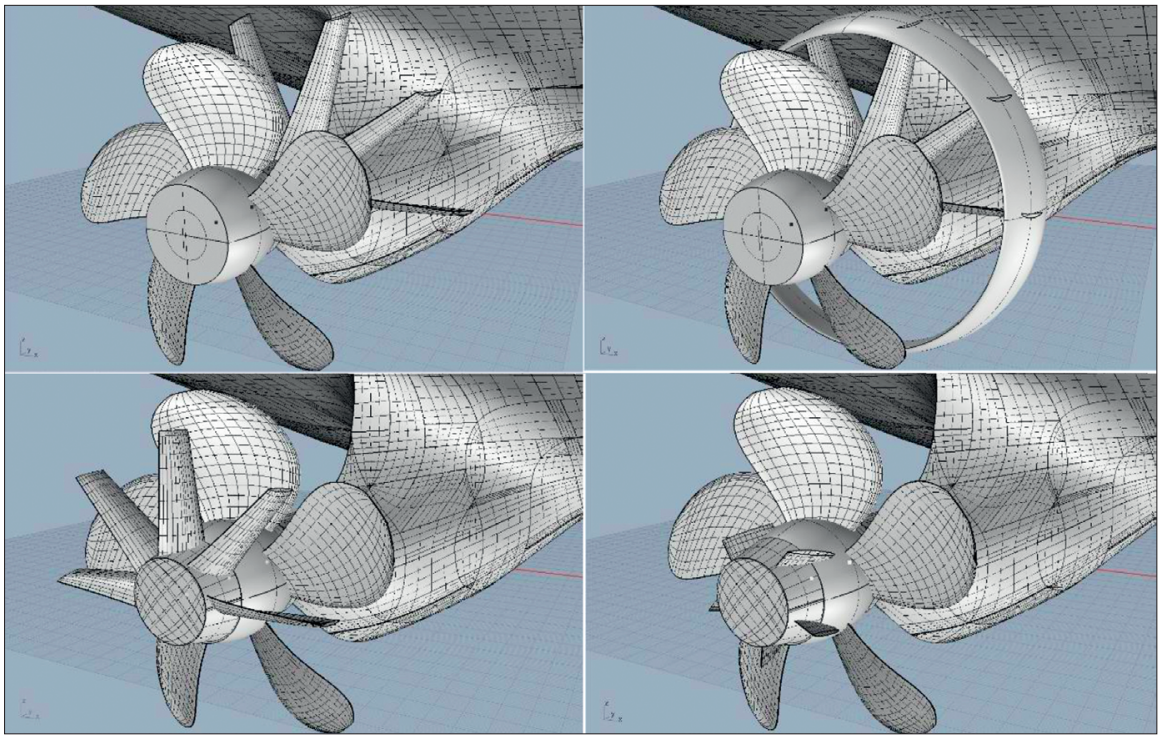

Fig. 1. Various kinds of ESDs (from upper left to bottom right): Pre-swirl stator, pre-swirl duct, post-swirl stator, propeller boss cap fins 
counter-swirl, reducing the tangential losses of the propeller energy. In this way the propeller efficiency is altered, but the foils generate drag on themselves.

The pre-swirl duct (PSD) is a system of PSS foils shrouded with the duct. It combines the advantages of the PSS with equalling the velocity field inflowing to the operating propeller, which may improve the propulsor power profile as well as its cavitation/vibratory characteristics.

The post-swirl stator (PoSS) is based on the opposite concept to the PSS; the foils are located after the propeller, in its slipstream. The stator recovers energy lost by the propeller due to tangential losses by generating the thrust on its blades.

The propeller boss cap fins (PBCF) are a set of small lifting foils, located downstream from the propeller's trailing edge on the hub itself. Their role is to dispel the hub vortex shedding from the propeller and to recover some of the tangential losses.

A kind of PoSS that is present on most ships is a rudder. Its displacement effect is normally beneficial for the propeller by a slight increase of the effective wake fraction. Moreover, it may have a twisted leading edge and generate additional thrust, recovering the propeller's tangential losses.

This paper is divided into three parts: the first sketches the most important features of design methods met in the literature. The fluid flow models employed are named and ESD design criteria are mentioned. It should, however, be noted that the vast majority of modern studies adopt CFD computations combined with variational optimization of the parametrically described ESD.

The second, main, part of the paper summarises the most important results reported in each article. In the majority of studies, both numerical and experimental data were available, in which case the numerical data are mostly omitted in this review, due to space limitations.

The last, concluding, part of this review presents and compares the results reported in selected papers. In fact 'selected papers' means here 'all those for which it was possible'. This is due to the huge variety of data presented in the literature and difficulties with constructing a common base.

\section{THE DESIGN METHODS}

The stand-alone screw propeller has huge variety of wellrecognised methods. This is somewhat contradictory to the propeller-ESD propulsor system, for which, due to the high complexity of the flow phenomena in such system, many more methods exist in the literature. None of them, however, can be stated as canonical or as reliable as classical approaches for a propeller operating alone. The reported methods can be distinguished mainly by the flow representation employed; it can be either an inviscid vortex flow model or a viscous one. A second factor is the stator particular that is undergoing the design process: it can be either the bound circulation distribution (in which case the stator foil's geometry results directly from it) or the geometry. In the latter case, the information on bound circulation is most often omitted in
Tab. 1. Design approach in selected papers

\begin{tabular}{|c|c|c|c|}
\hline Paper & $\begin{array}{l}\text { Designed } \\
\text { particular }\end{array}$ & $\begin{array}{l}\text { Design } \\
\text { criterion }\end{array}$ & $\begin{array}{l}\text { Flow } \\
\text { model }\end{array}$ \\
\hline$[2]$ & Circulation distribution & Rotation cancellation & Vortex \\
\hline [3] & Geometry & Optimization & Viscous \\
\hline$[4]$ & Circulation distribution & Optimization & Vortex \\
\hline$[5]$ & Circulation distribution & Optimization & Vortex \\
\hline [7] & Circulation distribution & Optimization & Vortex \\
\hline$[8]$ & Circulation distribution & Rotation cancellation & Vortex \\
\hline [9] & Geometry & Optimization & Viscous \\
\hline$[10]$ & Geometry & Optimization & Viscous \\
\hline$[11]$ & Circulation distribution & Optimization & Vortex \\
\hline$[12]$ & Geometry & Optimization & Viscous \\
\hline$[13]$ & Circulation distribution & Optimization & Vortex \\
\hline$[14]$ & Geometry & Optimization & Viscous \\
\hline$[15]$ & Geometry & Optimization & Viscous \\
\hline$[16]$ & Circulation distribution & Optimization & Vortex \\
\hline$[21]$ & Geometry & Optimization & Viscous \\
\hline$[22]$ & Geometry & Optimization & Viscous \\
\hline$[27]$ & Geometry & Optimization & Viscous \\
\hline$[29]$ & Geometry & Streamline adjustment & Viscous \\
\hline$[31]$ & Geometry & Optimization & Vortex \\
\hline$[32]$ & Circulation distribution & $\mathrm{n} / \mathrm{d}$ & Viscous \\
\hline
\end{tabular}

the papers and it is in fact the only design concept available for the RANSE-based approach.

Among the vortex models, the most basic tool is the lifting line model (LLM), replacing stator foils with straight line bound vortices. This is a sufficient simplification for determination of the induced tangential velocity coming from the stator, which is in fact the vital part from the cooperating propeller point of view. In such approach, stator vortex wake deformation can be taken into account, which allows more accurate determination of the stator-induced axial velocity component [17]. Stators, with foils of high aspect ratio, may also be successfully designed with the LLM. Such approach seems, however, to be highly outmoded nowadays, as already in 1988 Kerwin used the lifting surface model (LLS) for this task [11]. The most advanced vortex flow model, the boundary element method (BEM), cannot be directly utilised in the design of the foil geometry for the prescribed loading. Thus it is rather addressed in the design via variational geometry optimization and/or analysis of the given propulsor system geometry. The decision on the stator's bound circulation magnitude is crucial for the efficiency of the propulsor system. Due to the lack of a theoretical criterion, variational optimization is done in most cases.

Vortex methods have several advantages which encourage many designers to use them, especially at the initial stages of work. The easy individual code implementation, 
relatively short calculation time and the possibility of reverse problem solution are the main advantages of these. There are, however, serious limitations also. Computation of the vortex wake deformation, which could affect the designed foil's geometry, requires much care and may pose a difficulty in the case of heavily loaded stators, especially if the propeller-induced velocity field is to be taken into account during the simulation. Moreover, there are certain phenomena, like flow separation and vorticity dissipation, that cannot be included directly within the vortex model and have to be handled with semi-empirical formulas or in some other way. Flow separation is likely to occur on highly loaded profiles. Within the frame of the vortex model one can design a section with an arbitrary lift magnitude and distribution (even for NACAa $=1.0$ mean line), but exceeding reasonable values will result in a failure to achieve the assumed loading in real fluid. Finding that limit may be done based upon systematic blade section profiles data, as collected in [1], and control of the lift force coefficient [7] or angle of attack [14] values.

Once the viscous flow model is adopted, most of the mentioned difficulties are driven away; the vortex wake shape, separation and vorticity dissipation are included directly in the simulation. Meanwhile, however, also all the advantages favouring vortex models are gone. Viscous calculations require notable computer resources and - what is most problematic - reverse problem solution is no longer possible. Instead, one has to employ optimization of both the stator and propeller geometries for the prescribed operating conditions. This may notably increase the required time of realization. Thus, optimization is required not only for the ESD geometry undergoing the design process but for the computational mesh also, to provide a sufficient balance between accuracy and the computational cost [15].

Despite using a much more sophisticated approach, RANSE can still provide only limited accuracy. A model test prognosis for an accelerating duct influence of $6.03 \%$ was already considered to be in sufficient agreement with CFD calculations, indicating a 5.15\% reduction [3]. Similarly, for the pre-swirl stator a $5.2 \%$ power reduction, indicated by CFD, was outweighed by sea trials results of $6.8 \%$ [31]. This situation results probably from inaccuracies of local vortex phenomena modelling, which are responsible for an energy recovery effect. The accuracy may be improved if the relatively simpler turbulence model (e.g. k- $\omega$ SST) is replaced with the more sophisticated 7-equation RST model; the propellerdelivered power error may be decreased from around $4.0 \%$ to even $1.3 \%$ [15]. In the case of unsteady simulations, the time step turns out also to have vital importance [25], [26]. For this reason, new efforts on CFD development are currently being made, with self-propulsion conditions as a research topic [30], [33].

Correct modelling of such systems operation requires reference data for simulation validation, not only for propulsor operation but for the velocity fields also, such as is gathered in [6].
From a practical point of view, especially interesting is conducting the CFD calculations in full scale [15], [27], [31], although model-scale calculations are being conducted nowadays also [3], [12]. Both approaches have their advantages. One of the more important, shared by both, is getting a better insight into the complex flow phenomena involved in ESD operation [7], [12]. The main weight of interest remains in the velocity field and its alteration by ESD installation [28]. An important research field, however, is cavitation simulation [34].

Due to the issues outlined above, the most reasonable approach seems to be to start the design process with a vortex model to determine the initial geometry of the system (as shown e.g. in [9]). Once this is known, the optimization may be finalized by CFD simulation, conducted for a narrower number of cases.

\section{ENERGY-SAVING EFFECT}

A considerable number of papers upon the propeller-stator topic have been published, most of them reporting on the power reduction delivered, as provided by the ESD of the authors' design. The influence of the ESD is determined by means of either numerical calculations or model tests; in some cases, sea trials results are even provided. The completeness of the available data differs widely; in some cases not even the propeller diameter or ship speed are provided. This makes comprehensive analysis more difficult and less detailed than one would wish. In most papers, both numerical and experimental results are available. In such cases, only empirical data are repeated below, as the main focus of this study is ESD operation, not CFD development. Due to the requirements of further analyses, standard propeller loading coefficients like $K_{T}, K_{Q}$ and $C_{T}, C_{N}$ values were calculated (if these were not provided), based upon the data provided in the original papers.

$$
\begin{array}{cc}
K_{T}=\frac{T}{\rho n^{2} D^{4}} & K_{Q}=\frac{Q}{\rho n^{2} D^{5}} \\
C_{T}=\frac{T}{\frac{1}{8} \pi \rho D^{2} V^{2}} & C_{N}=\frac{2 \pi n Q}{\frac{1}{8} \pi \rho D^{2} V^{3}}
\end{array}
$$

After some considerations, an effective advance speed for calculation of $C_{T}, C_{N}$ was calculated using the advance ratio/ effective wake coefficient determined with the ESD installed (if such value was available). Such approach was adopted due to the author's feeling that it better reflects propeller loading conditions, even if is not formally equal to the mean axial velocity experienced by operating the propeller. Unless stated otherwise, water density was taken as $\rho_{M}=998.6 \mathrm{~kg} / \mathrm{m}^{3}$ for the model scale and $\rho_{S}=1025.9 \mathrm{~kg} / \mathrm{m}^{3}$ for full scale.

The authors of each study adopted various vessels for their analyses. The main particulars of these are summarised in Table 2. A blank space was left if some data were not provided. 
Tab. 2. Vessels analysed in particular studies

\begin{tabular}{|c|c|c|c|c|c|c|c|}
\hline Paper & Ship type / Name & $\mathrm{L}_{\mathrm{pp}}[\mathrm{m}]$ & $B[\mathrm{~m}]$ & $\mathrm{T}[\mathrm{m}]$ & $\mathrm{C}_{\mathrm{B}}[-]$ & $\mathrm{D}[\mathrm{m}]$ & $\mathrm{V}_{\mathrm{DES}}[\mathrm{knots}]$ \\
\hline [2] & Trawler & & & & & & \\
\hline [2] & Tanker & & & & & & \\
\hline [3] & Japanese Bulk Carrier (JSBC) & 280.0 & 45.00 & 16.5 & & 8.12 & 7.5 \\
\hline$[3]$ & Tanker & 333.0 & 60.0 & 20.5 & & 10.5 & \\
\hline [4] & Cargo Vessels & & & & & & \\
\hline [5] & None & & & & & & \\
\hline [7] & Container Vessel & & & & & 8.75 & \\
\hline [8] & Container Ship & 286.0 & 48.20 & 14.8 & & 9.60 & 22.0 \\
\hline [9] & VLCC & 322.0 & 60.00 & 21.0 & & 10.00 & \\
\hline [10], [24], [32] & Kriso Container Ship (KCS) & 230.0 & 32.20 & 10.8 & 0.651 & & 24.0 \\
\hline$[12]$ & 13,200 TEU Container Ship & 350.0 & 48.2 & 14.5 & & & 20.0 \\
\hline [13] & $\mathrm{n} / \mathrm{a}$ & 319.0 & 60.00 & 21.0 & & 10.00 & 16.21 \\
\hline$[14]$ & KVLLC & 320.0 & 58.00 & 20.8 & 0.810 & & 15.5 \\
\hline$[15]$ & Chemical Tanker & 175.6 & 32.23 & 11.85 & 0.812 & 6.50 & 14.0 \\
\hline [17] & Nawigator XXI & 54.13 & 10.5 & $3.15 / 3.20^{\mathrm{a}}$ & 0.626 & 2.26 & 13.0 \\
\hline [21] & $\mathrm{n} / \mathrm{a}$ & 174 & 32.20 & 11 & & 6.10 & 15 \\
\hline [22] & 6,500 TEU Container Ship & & & & & & \\
\hline [24] & KVLLC2 & & & & & & 15.5 \\
\hline [27] & 32500 DWT Bulk Carrier & 171.5 & 28.40 & 10.0 & & 5.20 & $11.5 / 12.0$ \\
\hline [29] & 180m Diamond 34 Bulk Carrier & 176.8 & 30.00 & 9.75 & 0.800 & 5.60 & 14 \\
\hline$[21]$ & Bulk Carrier "VALVOLINE" & 182.0 & 32.00 & 11 & & 5.80 & 15.0 \\
\hline
\end{tabular}

a - given as $T_{A} / T_{F}$

\section{UPSTREAM DEVICES}

The main attention of this review is to the upstream ESDs. Table 3 (next page) summarises the results of the model tests and calculations conducted in each study. Where it was possible, the data for 'no ESD' conditions are provided here also. For such cases, a comment 'none' is put in the 'ESD type' column. The power-saving effect $\Delta P_{D}$ is defined according to the formula:

$$
\Delta P_{D}=\frac{P_{D}^{N o E S D}-P_{D}^{E S D}}{P_{D}^{N o E S D}}
$$

Selected papers are briefly discussed below. The paper [24] covers a vital topic; it is focused not on the design process itself but on the method of model tests extrapolation for the vessels with pre-swirl ESDs. The main issue is in fact the extrapolation of the effective wake fraction. The authors analyse the results of adopting known approaches (ITTC'78, ITTC'99) and their own. The main difference between the ITTC methods and the proposed one includes the fact that the pre-swirl devices not only produce rotational inflow but also alter the axial inflow, which should be treated separately during scaling. A wide range of model test results are given in the paper. Two vessels are taken into analysis: KVLLC2 (equipped with a pre-swirl duct) and KCS (equipped with a pre-swirl stator) and results of adopting the subsequent full-scale prognosis methods are compared.
Tab. 4. Full-scale propulsion prognosis via subsequent methods

\begin{tabular}{|c|c|c|c|c|c|c|}
\hline $\begin{array}{c}\text { Scaling } \\
\text { method }\end{array}$ & ESD & Vessel & $\begin{array}{c}V_{S} \\
{[\mathrm{knots}]}\end{array}$ & $\begin{array}{c}w_{E S} \\
{[-]}\end{array}$ & $\begin{array}{c}P_{D S} \\
{[\mathrm{~kW}]}\end{array}$ & $\begin{array}{c}n_{S} \\
{[\mathrm{rpm}]}\end{array}$ \\
\hline ITTC'78 & None & KVLLC2 & 15.5 & 0.342 & 26226 & 71.73 \\
\hline ITTC'78 & PSD & KVLLC2 & 15.5 & 0.363 & 24959 & 70.42 \\
\hline TTC'99 & PSD & KVLLC2 & 15.5 & 0.383 & 24384 & 69.53 \\
\hline $\begin{array}{c}\text { Moon et al., } \\
\text { 2017 }\end{array}$ & PSD & KVLLC2 & 15.5 & 0.367 & 24793 & 70.23 \\
\hline $\begin{array}{c}\text { ITTC'78 } \\
\text { ITTC'78 }\end{array}$ & None & KCS & 24.0 & 0.254 & 43.672 & 106.28 \\
\hline $\begin{array}{c}\text { ITTC'99 } \\
\text { TCS }\end{array}$ & PSS & KCS & 24.0 & 0.282 & 42.857 & 105.19 \\
\hline $\begin{array}{c}\text { Moon et al., } \\
2017\end{array}$ & PSS & KCS & 24.0 & 0.298 & 42.094 & 104.11 \\
\hline
\end{tabular}

Paper [9] starts with an analysis of the energy losses occurring on the marine propeller, providing vital insight into possible recovery concepts. Axial losses are deduced via ideal propulsor theory. Rotational losses were taken as a remaining factor between the real propeller efficiency and the $\eta i$ determined via BEM, with no viscosity effects included.

The design of a pre-swirl duct was realised via variational optimization by means of FVM simulation. The vessel adopted for the design example was VLCC. The model tests were conducted both for 'bare hull' conditions and with ESD installed. The precise value of the delivered power prognosis was not given in the paper; only a quasi-propulsive efficiency increase of $5.1 \%$ was declared. This is at a similar level to the 
Tab. 3. Upstream device installation effect

\begin{tabular}{|c|c|c|c|c|c|c|c|c|c|}
\hline Paper & Ship type / Name & ESD type & $\Delta P_{D}[\%]$ & $V_{s}[\mathrm{kn}]$ & $\mathrm{n}[\mathrm{rpm}]$ & $K_{T}[-]$ & $10 K_{Q}[-]$ & $C_{T}[-]$ & $C_{N}[-]$ \\
\hline [3] & JSBC & $\mathrm{AD}$ & 6.0 & 7.5 & & & & & \\
\hline$[3]$ & Tanker & PSD & 3.5 & 8.1 & & & & & \\
\hline [5] & None & none & - & & & 0.215 & 0.389 & 0.691 & 0.884 \\
\hline$[5]$ & None & PSS & 3.0 & & & 0.215 & 0.375 & 0.689 & 0.850 \\
\hline [7] & Container Vessel & none & - & & 100.2 & & & & \\
\hline [7] & Container Vessel & PSS & 5.0 & & 96.0 & & & & \\
\hline [9] & VLCC & none & - & & & 0.163 & & 2.050 & \\
\hline [9] & VLCC & PSD & 5.1 & & & 0.186 & & 2.952 & \\
\hline$[10]$ & KCS & none & - & 24.0 & $654.0^{\mathrm{M}}$ & & & & \\
\hline$[10]$ & KCS & PSS & 3.9 & 24.0 & $639.0^{\mathrm{M}}$ & & & & \\
\hline$[13]$ & $\mathrm{n} / \mathrm{a}$ & none & - & 16.21 & $473.4^{\mathrm{M}}$ & 0.177 & 0.200 & 2.753 & 4.860 \\
\hline$[13]$ & $\mathrm{n} / \mathrm{a}$ & PSS & 2.5 & 16.21 & $468.6^{\mathrm{M}}$ & 0.181 & 0.201 & 3.284 & 6.050 \\
\hline$[13]$ & $\mathrm{n} / \mathrm{a}$ & PSD & 5.8 & 16.21 & $459.0^{\mathrm{M}}$ & 0.179 & 0.195 & 3.639 & 6.805 \\
\hline [14] & KVLLC & none & - & 15.5 & 75.1 & & & & \\
\hline$[14]$ & KVLLC & PSS & 5.6 & 15.5 & 70.9 & & & & \\
\hline [15] & Chemical Tanker & none & - & 14.0 & 86.4 & & & & \\
\hline$[15]$ & Chemical Tanker & PSS & & 14.0 & & & & 1.570 & 2.703 \\
\hline [17] & Nawigator XXI & none & - & 13.0 & 283.3 & 0.199 & 0.264 & 3.236 & 6.798 \\
\hline$[17]$ & Nawigator XXI & PSS & 10.0 & 13.0 & 273.6 & 0.225 & 0.283 & 4.890 & 11.258 \\
\hline$[17]$ & Nawigator XXI & none & - & 13.0 & 269.8 & 0.231 & 0.308 & 3.226 & 6.335 \\
\hline$[17]$ & Nawigator XXI & PSS & 7.4 & 13.0 & 257.7 & 0.255 & 0.327 & 4.825 & 10.589 \\
\hline$[21]$ & $\mathrm{n} / \mathrm{a}$ & none & - & 15.0 & & & & & 3.698 \\
\hline$[21]$ & $\mathrm{n} / \mathrm{a}$ & PSD & 3.3 & 15.0 & & & & & 5.716 \\
\hline$[24]$ & KVLLC2 & none & - & 15.5 & 71.7 & & & & \\
\hline$[24]$ & KVLLC2 & PSS & 3.1 & 15.5 & 69.5 & & & & \\
\hline$[24]$ & KCS & none & - & 24.0 & 106.3 & & & & \\
\hline$[24]$ & KCS & PSS & 4.2 & 24.0 & 103.7 & & & & \\
\hline$[27]$ & 32500 DWT Bulk Carrier & none & - & 11.5 & $499.7^{\mathrm{M}}$ & & 0.196 & & 5.858 \\
\hline [27] & 32500 DWT Bulk Carrier & PSS & 2.5 & 11.5 & $486.2^{\mathrm{M}}$ & & 0.208 & & 8.915 \\
\hline [29] & 180m Diamond 34 Bulk Carrier & none & - & 14.0 & $559.2^{\mathrm{M}}$ & & 0.207 & & \\
\hline [29] & 180m Diamond 34 Bulk Carrier & PSS & 1.6 & 14.0 & $550.2^{\mathrm{M}}$ & & 0.214 & & \\
\hline$[31]$ & Bulk Carrier "VALVOLINE" & PSS & 6.8 & 15.0 & 123.0 & & & & \\
\hline
\end{tabular}

AD - accelerating duct; PSD - pre-swirl duct; PSS - pre-swirl stator; ${ }^{\mathrm{M}}$ - model-scale value

Tab. 5. Particulars of analysed propeller and its operation

\begin{tabular}{|c|c|c|c|c|c|c|c|c|c|}
\hline$D[\mathrm{~m}]$ & $P_{0.7} / D[-]$ & $A_{E} / \mathrm{A}_{0}[-]$ & $Z[-]$ & $Z[-]$ & $K_{T}[-]$ & $\eta_{i}[-]$ & Axial losses [-] & Rotational losses [-] & $C_{T^{a}}[-]$ \\
\hline \multirow{3}{*}{10.0} & \multirow{3}{*}{0.714} & \multirow{3}{*}{0.4} & \multirow{3}{*}{4} & 0.40 & 0.1855 & 0.6038 & 0.3308 & 0.0355 & 2.952 \\
\cline { 5 - 11 } & & & & 0.45 & 0.1630 & 0.6675 & 0.2718 & 0.0312 & 2.050 \\
\cline { 5 - 11 } & & & & 0.50 & 0.1401 & 0.7285 & 0.2183 & 0.0268 & 1.427 \\
\hline
\end{tabular}

a - calculated based on data provided in original paper

one reported in paper [13], where a power reduction at a level of $5.8 \%$ was declared from PSD installation (versus $2.5 \%$ from PSS application). The latter paper also provides detailed results of the model tests.

In paper [21] a 'Crown Duct' (CD) ESD device is discussed. Unlike in the case of a 'casual' PSD, the duct part is not located at the ESD's tip but rather in midspan. Some of the pre-swirl foils end at the duct, but some of them extend beyond it or even start at it. The CD design is realised via variational optimization by means of FVM simulation. For the design example, a vessel of design speed $V_{S}=15$ knots is adopted. The model tests were carried out both for a 'bare' hull and with CD installed, for two draughts ( $T_{A}, T_{F}$ values were not precisely defined). For full-scale prediction, the ITTC'99 procedure of effective wake 
Tab. 6. Full-scale prognosis

\begin{tabular}{|c|c|c|c|c|c|c|c|c|c|}
\hline$V_{S}=13.0$ knots & $J_{S}[-]$ & $w_{T S}[-]$ & $\eta_{H S}[-]$ & $\eta_{0 S}[-]$ & $\eta_{D S}[-]$ & TS $[\mathrm{kN}]$ & $Q_{S}[\mathrm{kNm}]$ & $n_{s}[\mathrm{rpm}]$ & $P_{D S}[\mathrm{~kW}]$ \\
\hline CP745 & 0.396 & 0.371 & 1.221 & 0.444 & 0.566 & 124 & 37.1 & 289.3 & 1123 \\
\hline $\mathrm{CP} 745+\mathrm{ST} 001^{\mathrm{a}}$ & 0.342 & 0.473 & 1.444 & 0.410 & 0.626 & 125 & 35.4 & 273.6 & 1014 \\
\hline CP745+ST001 ${ }^{\mathrm{b}}$ & 0.340 & 0.476 & 1.452 & 0.408 & 0.627 & 125 & 35.4 & 273.0 & 1011 \\
\hline CP753 & 0.427 & 0.351 & 1.191 & 0.497 & 0.597 & 125 & 37.7 & 269.8 & 1065 \\
\hline CP753+ST002 & 0.367 & 0.468 & 1.419 & 0.438 & 0.645 & 126 & 36.5 & 257.7 & 986 \\
\hline $\mathrm{CP} 753+\mathrm{ST} 002^{\mathrm{b}}$ & 0.363 & 0.474 & 1.435 & 0.434 & 0.646 & 126 & 36.5 & 257.0 & 982 \\
\hline
\end{tabular}

a - scaling method according to ITTC'99; b - scaling method proposed in (Moon-Chan et al., 2017)

coefficient scaling was adopted and a power saving of $3.3 \%$ was declared. The paper gives detailed results of the model tests.

Paper [3] begins with presentation of the data for a validation case, which is a Japanese Bulk Carrier (JBC). For a speed around $V_{S}=7.5$ knots $(F r=0.142$, as given in the paper), the model test prognosis for delivered power is $P_{D S}=2863 \mathrm{~kW}$. The corresponding test after installing the ESD (an accelerating duct) yielded a delivered power prognosis of $P_{D S}=2691 \mathrm{~kW}$. The resulting power reduction of $6.03 \%$ was considered to be in sufficient agreement with one determined via finite volume calculations (5.15\%). FVM simulation was applied to design via optimization the geometry of the ESD for another vessel, the Tanker. For a speed around 8.1 knots $(F r=0.141$, as given in the paper), the model test prediction for the delivered power is $P_{D S}=2813 \mathrm{~kW}$. The corresponding test after installing the ESD (a pre-swirl duct) brought a delivered power prediction of $P_{D S}=2716 \mathrm{~kW}$. The resulting power reduction of $3.45 \%$ is closer to the one determined via FVM simulation (2.95\%) than in the case of the JBC.

In paper [7] the pre-swirl stator is designed via the lifting line method, which is applied for optimization of the bound circulation distribution. For avoidance of flow separation on the stator, the local value of the lift coefficient $C_{L}$ is kept below the prescribed margin (the precise value is not given in the paper). For the design case, a container vessel was adopted (the vessel particulars are not provided, the propeller diameter is $D=8.75 \mathrm{~m}$ ). The model tests were conducted for a pre- swirl stator of diameter $2 \Lambda=9.10 \mathrm{~m}$, and two propellers revealed a possible delivered power reduction at a level of $5.0 \%$ along with a rate of revolution reduction by $4.2 \%$. A quite similar concept is given in paper [14], where, however, the stator design is realised via the lifting surface method. It is assumed to cancel around $50 \%$ of the propeller slipstream rotation and have elliptic loading on each blade. Flow separation is avoided by limitation of the angle of attack to $15^{\circ}$. For the design example, the KVLLC with a design speed of $V_{S}=15.5$ knots was adopted. The model tests were conducted for three configurations: without any ESD installed, with PSS as designed and with the same PSS but turned by $180^{\circ}$. The tests indicated a power reduction at a level of $5.6 \%$ and a propeller rate of revolution reduction at a level of $5.5 \%$. Meanwhile, the mirror PSS kept a very similar revolution reduction but nearly no power reduction $(0.7 \%)$.

Paper [27] addresses the topic of a PSS in the form of controllable pre-swirl fins (CPSF), having fixed and controllable ('flap') parts. The blades are designed via variational optimization by means of FVM simulations. The advantage of the adopted solution is that each blade can be adapted for actual vessel loading conditions to preserve the optimal propulsor operation. For the design example, a 32500 DWT bulk carrier was selected. The model tests were carried out for two draughts: 'Design' ( $T=10.0 \mathrm{~m}$, even keel) and 'Ballast' $\left(T_{A}=7.0 \mathrm{~m}, T_{F}=5.0\right)$, both for the propeller operating alone and with ESD installed, at each draught. The flap angle was optimised before these tests. Power saving at a level of $2.5 \%$ for the design draught and even $4.8 \%$ for the ballast draught was declared.

Paper [15] addresses the topic of pre-swirl stator design. As in most cases in current research, it is realised via optimization by means of FVM simulation. The vessel adopted for the design example is the Chemical Tanker. Despite the model tests conducted, no complete full-scale prognosis with the ESD is provided in the paper. Only selected propulsive coefficients are compared before and after installing the ESD, and it can be stated only that the expected hull efficiency value will rise from the value of 1.170 to 1.242 due to the presence of the ESD.

In the dissertation [17], two propulsor systems are presented (along with model test results): propeller CP745 with the pre-swirl stator ST001 and propeller CP753 with the PSS ST002. Both propulsor systems were designed via a combined lifting line-lifting surface approach (the latter with its more mature version). Each PSS blade is assumed to have an elliptic loading distribution. The vessel adopted for the design case was Nawigator XXI with the design speed defined as $V_{S}=13.0$ knots. The model tests were conducted for cases both with and without the ESD.

The discussed propulsor systems were earlier presented in papers [16], [18], [19] and [20]. The data presented in [17] may differ slightly as it was re-calculated anew.

Paper [31] describes the PSS design procedure and analysis of its impact on the economic aspect of the vessel's exploitation. As stated in the paper, the design procedure includes:

"1. RANS computation to obtain the wake field at the pre-selected PSS positions

2. BEM optimisation to obtain the optimal twist and camber of the PSS

3. Creation of parametric model for ESD

4. RANS Self-propulsion computations/optimisations to evaluate the PSS design". 
For the design example, the Bulk Carrier "VALVOLINE" with the design speed of $V_{S}=15.0$ knots was adopted. The design propeller rate of revolution is defined as $n_{S}=123 \mathrm{rpm}$. The FVM simulation predicted a delivered power reduction by $5.3 \%$ due to PSS installation, while sea trials revealed it to be $6.8 \%$. The propeller rate of revolution was reduced by $5.2 \%$ due to the PSS.

In paper [32] the approach to PSS design is based on circulation distribution, replaced with the equivalent angle of attack. The detailed criteria for selecting the stator loading distribution and magnitude are not given, however. For the design example, the Kriso Container Ship (KCS) was adopted. The numerical results presented in the paper refer to the influence of the PSS on the hull resistance (resistance tests, without operating propeller), indicating a predicted effective power increase of $11 \%$ due to installation of the PSS. No self-propulsion test results or simulations were presented in this paper.

\section{DOWNSTREAM DEVICES}

Table 7 is constructed in a similar manner to Table 3 and presents corresponding data for downstream devices.

Paper [2] covers the topic of a post-swirl stator. Vortex representation of the lifting blades is utilised. The propeller is designed individually and then the stator is designed as a retrofit and the study "therefore neglects the effect of the stator in the optimization process of the propeller". The design criterion for the stator demands cancellation of the fluid rotation passing through it. This criterion gives a direct relation between the bound circulation on the stator and that of the propeller. Two design examples are presented in the paper, the 'Trawler' and the 'Tanker'. The particulars of the vessels are not provided; only operating parameters assumptions are given. An FVM simulation for the full scale was carried out to analyse the designed propulsors (propeller + stator). A similar design approach to PoSS is adopted in paper [8]. A vortex model is applied and the design criterion demands rotation cancellation after the stator also. As the design example a $286 \mathrm{~m}$ Container Ship was adopted. The required thrust is defined as "roughly $T=2910416.35 N$ ". The model tests indicate $6.1 \%$ point higher efficiency for the propeller+stator system than for the equivalent stand-alone propeller.

In paper [10] the topic is a combined ESD, consisting of a wavy twisted rudder (WTR), tip raked propeller (TRP) and pre-swirl stator. The PSS design is conducted via potential code in the initial stage and is finalised with FVM. The design case adopted as the example is the Kriso Container Ship (KCS). In the first part of the paper, model tests with various rudder types are conducted to reveal the delivered power's dependency on the selected rudder type. Based upon these results, the WTR was adopted for further work. Subsequent model tests were conducted with each ESD (PSS, TPR and WTR) installed alone and with all of them together (combined ESD), revealing that the sum of the power-saving effects of ESDs installed individually (7.16\%) is notably higher than that for the combined ESD (5.36\%). This is easily explained by the fact that some of the applied ESDs recover the same energy losses components.

Paper [12] covers the topic of twisted rudder design. Three types of twisted rudders were investigated in this study, including a 'bare' twisted rudder blade, a second one with a bulb and a third with additional fins installed on the bulb. As a reference level, a standard horn-type rudder was taken. The model tests revealed that the rudder equipped with the bulb brought the lowest saving effect (2.0\%), while the one with additional fins on the bulb gave the highest effect (2.9\%). The bare twisted rudder gave a $2.3 \%$ power-saving effect.

Paper [29] is focused mainly on the rudder type and the influence of its distance from the propeller on the propulsion

Tab. 7. Effect of downstream device installation

\begin{tabular}{|c|c|c|c|c|c|c|c|c|c|}
\hline Paper & Ship type / Name & ESD type & $\Delta P_{D}[\%]$ & $V_{S}[\mathrm{kn}]$ & $\mathrm{n}[\mathrm{rpm}]$ & $K_{T}[-]$ & $10 K_{Q}[-]$ & $C_{T}[-]$ & $C_{N}[-]$ \\
\hline$[2]$ & Trawler & PoSS & & 10.0 & 380.0 & 0.104 & 0.117 & 2.792 & 6.388 \\
\hline$[2]$ & Tanker & PoSs & & 12.5 & 247.0 & 0.181 & 0.232 & 2.294 & 4.119 \\
\hline$[4]$ & Cargo Vessel A & PBCF & 0.8 & & & 0.388 & 0.475 & 5.105 & 8.921 \\
\hline [4] & Cargo Vessel B & $\mathrm{PBCF}$ & 1.8 & & & 0.385 & 0.475 & 5.058 & 8.923 \\
\hline$[8]$ & Container Ship & none & - & 22.0 & & 0.220 & 0.344 & 1.073 & 1.458 \\
\hline$[8]$ & Container Ship & PoSS & 6.1 & 22.0 & & 0.239 & 0.345 & 1.166 & 1.463 \\
\hline$[10]$ & $\mathrm{KCS}$ & FSR & - & 24.0 & $654.0^{\mathrm{M}}$ & & & & \\
\hline$[10]$ & KCS & TR & 1.5 & 24.0 & $654.0^{\mathrm{M}}$ & & & & \\
\hline$[10]$ & KCS & WTR & 1.8 & 24.0 & $652.2^{\mathrm{M}}$ & & & & \\
\hline$[12]$ & 13,200 TEU Container Ship & none & - & 20.0 & $477.0^{\mathrm{M}}$ & 0.205 & 0.353 & 1.115 & 1.770 \\
\hline$[12]$ & 13,200 TEU Container Ship & ZB-F TR & 2.9 & 20.0 & $473.4^{\mathrm{M}}$ & 0.205 & 0.351 & 1.117 & 1.761 \\
\hline [29] & 180m Diamond 34 Bulk Carrier & Naca & 0.3 & 14.0 & 557.4 & 0.157 & 0.209 & & \\
\hline
\end{tabular}

PoSS - post-swirl stator; FSR - full spade rudder; TR - twisted rudder; WTR - wavy twisted rudder;

Orig - original horn rudder; Naca - spade rudder with NACA20 section; ZB-F TR - twisted rudder with bulb and fins; M - model-scale value 
characteristics. Moreover, the paper presents some results with the PSS installed; however, for this case only numerical results are available. For the design example a $180 \mathrm{~m}$ Diamond 34 Bulk Carrier was adopted. The design speed is $V=14$ knots $(F r=0.173)$. The model tests were conducted for two rudder types (ORIG - the original horn rudder and NACA - a spade rudder based on a NACA20 section), for three values of the propeller-rudder distance for each of them:

Tab. 8. Model test results for rudder ORIG

\begin{tabular}{|c|c|c|c|c|c|c|c|}
\hline Rudder & $\begin{array}{c}\Delta \mathrm{X} / \mathrm{D} \\
{[-]}\end{array}$ & $\begin{array}{c}T_{M} \\
{[\mathrm{~N}]}\end{array}$ & $\begin{array}{c}Q_{M} \\
{[\mathrm{Nm}]}\end{array}$ & $\begin{array}{c}n_{M} \\
{[\mathrm{rps}]}\end{array}$ & $\begin{array}{c}2 \pi \mathrm{Q}_{M} n_{M} \\
{[\mathrm{~W}]}\end{array}$ & $\begin{array}{c}K_{T^{a}} \\
{[-]}\end{array}$ & $\begin{array}{c}K_{\circ} a \\
{[-]}\end{array}$ \\
\hline ORIG & 0.455 & 42.78 & 1.333 & 9.42 & 78.92 & 0.1556 & 0.0205 \\
\hline ORIG & 0.371 & 42.22 & 1.318 & 9.32 & 77.18 & 0.1569 & 0.0208 \\
\hline ORIG & 0.286 & 42.97 & 1.331 & 9.32 & 77.96 & 0.1597 & 0.0210 \\
\hline NACA & 0.441 & 41.66 & 1.330 & 9.39 & 78.47 & 0.1525 & 0.0206 \\
\hline NACA & 0.357 & 41.75 & 1.323 & 9.35 & 77.68 & 0.1542 & 0.0207 \\
\hline NACA & 0.272 & 41.95 & 1.319 & 9.29 & 76.98 & 0.1569 & 0.0209 \\
\hline NACA & 0.272 & 41.95 & 1.319 & 9.29 & 76.98 & 0.1569 & 0.0209 \\
\hline
\end{tabular}

a - calculated based on data provided in original paper

The influence of the pre-swirl stator was analysed via FVM simulation only for configuration with the ORIG rudder. This simulation indicated a power reduction of $1.8 \%$ and a revolution reduction of $1.61 \%$ due to PSS installation. The detailed design procedure for the PSS was not provided in the paper. It was only stated that its blades are: "angled based on the flow direction in the region where they operate in order not to introduce extreme angles of attack and separation on the fins." Figures included in the paper suggest symmetric section profiles, but this is not stated directly. There must, however, be some non-zero camber and/or angle of attack, as there is a lift force generated on the PSS blades and it introduces the required counter-swirl, as stated in the paper.

In paper [4] a well-developed theoretical method for design of a propeller and dedicated propeller boss cap fins (PBCF) is provided. Preliminary calculations are based on vortex flow models, and precede more CFD calculations for verification of the produced design. In paper [22] PBCFs are analysed together with a divergent propeller hub cap. The first part of the paper is focused on representing the optimisation scheme, fuelled by CFD-derived and experimental data, where the parametrically described geometry of the PBCFs was analysed. A similar analysis was conducted for several concepts of the divergent hub cap. Another important contribution of this paper is the description of the experimental method, which is suitable for conducting an open water test for propellers equipped with PBCFs, which differs slightly from standard open water tests. The results show that the PBCFs may increase the propeller's open water efficiency, but the divergent cap applied for the propeller with PBCFs reduced the efficiency by $7.5 \%$, which suggests that this shape of cap was not good for this case. This conclusion is supported by the increased hub vortex reported for the model test with the divergent cap.

\section{PROPELLER LOADING AND ENERGY- SAVING EFFECT}

As one can imagine, the wide scatter of data presented above does not allow all of them to be arranged on a common base that would provide a reliable reference level for power-saving evaluation. Thus only selected values from the data presented above are taken for the analysis.

Plotting the energy-saving effect versus propeller loading (determined for 'No ESD' conditions), as in Fig. 2, leads to the clear conclusion that the propellers with higher loading reveal a higher potential for the energy-saving effect:

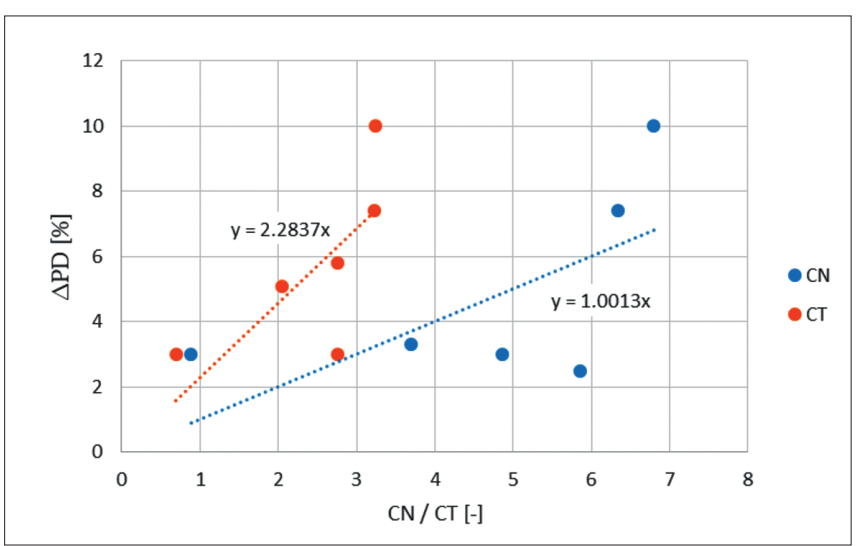

Fig. 2. Stand-alone propeller loading versus power-saving effect

Loading coefficients $C_{T}$ and $C_{N}$ represent the propeller loading with respect to the inflow velocity to the propeller disc. The propeller operating conditions may also be related to the rate of revolution, by coefficients $K_{T}, K_{Q}$. Plotting the energy-saving effect versus these, as in Fig. 3, did not allow any clear trend to be identified and hence no trend line was plotted.

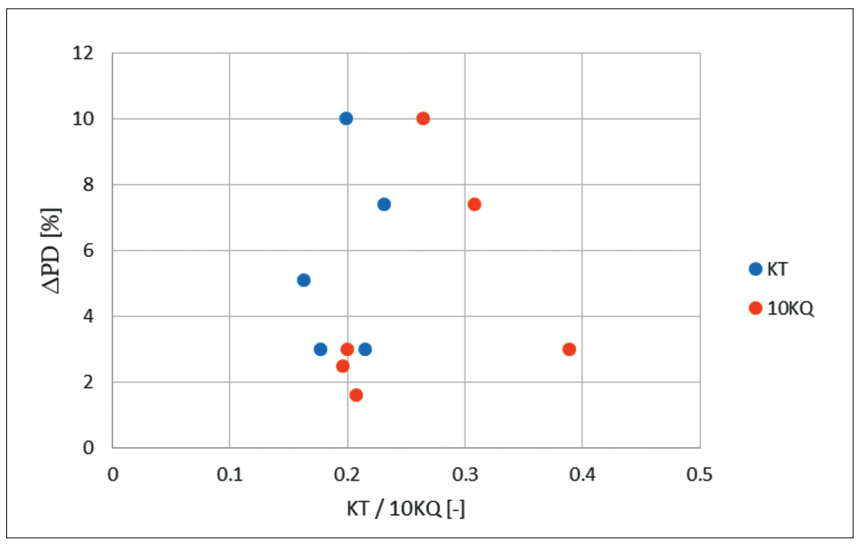

Fig. 3. Stand-alone propeller loading versus power-saving effect

The situation above is well explained by the fact that the $C_{T / N}$ coefficients relate the propeller loading to the energy brought by the inflowing stream, while $K_{T / Q}$ instead provides information on whether the propeller should be regarded as slow rotating or fast rotating. The latter concept is not necessarily directly determined by whether the propeller is highly or lightly loaded. 
Another possibility for evaluating the energy-saving effect is to plot it versus the revolution reduction, as presented in Fig. 4:

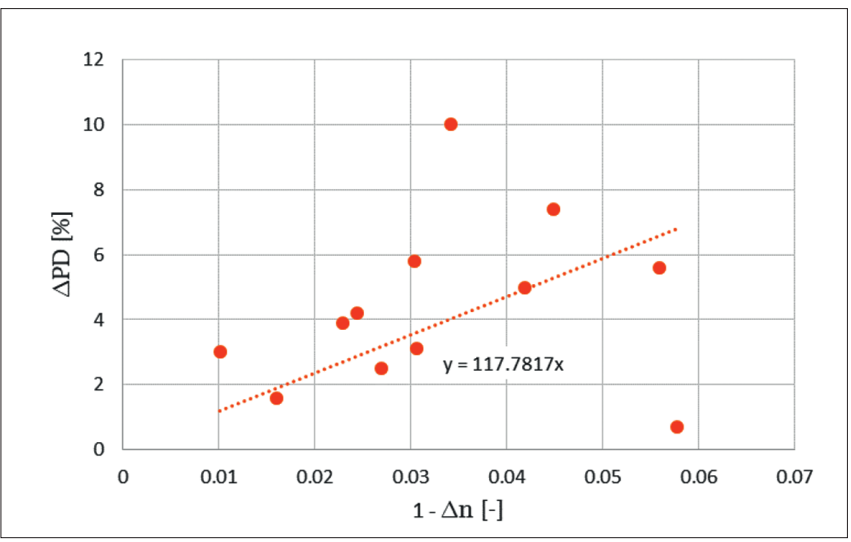

Fig. 4. Rate of revolution reduction versus power-saving effect

It has to be underlined that the latter method is not sufficient to evaluate the potential energy saving that can be expected for a particular propeller upon its stand-alone loading, but rather helps to evaluate the general quality of a particular ESD. A clear positive trend may be observed, but cases like the one described in [14] have to be kept in mind, when similar revolution reduction is accompanied by notably different power reduction values.

The analysis above was prepared for upstream devices only due to the completeness of the collected data. To evaluate the quality of the downstream devices analysed, their power savings were compared to the trend lines drawn for upstream devices:

Tab. 9. Downstream devices evaluation

\begin{tabular}{|c|c|c|c|c|c|c|}
\hline & & \multirow{2}{*}{ Paper } & \multirow{2}{*}{ Ship type / Name } & \multirow{2}{*}{ ESD } & \multicolumn{3}{|c|}{ Expected (trend line } & \multirow{2}{*}{$\begin{array}{c}\Delta P \\
\text { based on) }\end{array}$} \\
\cline { 4 - 6 } & & & $C_{T}$ & $C_{N}$ & $1-\Delta n$ & \\
\hline$[8]$ & Container Ship & PoSS & 2.5 & 1.5 & - & 6.1 \\
\hline$[10]$ & KCS & TR & - & - & 0.0 & 1.5 \\
\hline$[10]$ & KCS & WTR & - & - & 0.3 & 1.8 \\
\hline$[12]$ & $\begin{array}{c}13,200 \text { TEU } \\
\text { Container Ship }\end{array}$ & $\begin{array}{c}\text { ZB-F } \\
\text { TR }\end{array}$ & 2.5 & 1.8 & 0.9 & 2.9 \\
\hline$[29]$ & $\begin{array}{c}180 m \text { Diamond } \\
34 \text { Bulk Carrier }\end{array}$ & NACA & - & - & 0.4 & 0.3 \\
\hline
\end{tabular}

In table 9. signs “-” were put where sufficient data was not provided in the original papers. It can be seen, however, that the post-swirl stator reveals a notably more favourable operation effect than upstream devices installed with the propellers of corresponding loading. Another observation is that the trend line relating the power-saving effect with the revolution reduction may not be sufficient for evaluation of rudders that are considered as ESDs. It seems that the problem is not that there is no data for very low values of revolution reduction (the rudders analysed in this review yield nearly no revolution reduction), but the different hydrodynamic behaviour. Proper evaluation of rudders considered as ESDs requires a greater amount of data to be collected.

\section{CONCLUSIONS}

As concluding remarks of this review, the following may be stated:

- The design of ESDs is dominated by the use of optimization algorithms. The differences between particular studies lie mainly in the flow model adopted (potential or viscous) and the particular aspect undergoing the optimization process (loading distribution or geometry).

- Vortex models are being displaced by modern CFD but they still have an important role, especially at the initial stages of the design process. However, even modern CFD has a tendency to underestimate the expected power-saving effect.

- Proper analysis of vessels equipped with ESDs requires dedicated extrapolation methods for propulsion prognosis and sometimes conducting the model tests themselves.

- The expected power-saving level for most ESDs is around 3-6\%; higher propeller loading brings more potential for energy recovery. Each case should be analysed carefully to deduce the amount of energy losses that are to be recovered. - Among the upstream devices that were analysed in this review, pre-swirl ducts revealed power savings at a similar level to pre-swirl stators. However, their additional advantage is the improvement of the axial inflow to the propeller.

- Reasonable power saving may be achieved by replacing the classical spade rudder by one with a twisted leading edge (around 3\%).

\section{ACKNOWLEDGEMENT}

The research was partly financed by the National Centre for Research and Development of the Republic of Poland, within the framework of the Esthetics project (POLTUR3/ ESTHETICS/1/2019).

\section{REFERENCES}

1. I. H. Abbott, A. E. Doenhoff, Theory of wing sections including a summary of airfoil data. New York: Dover Publications, 1959.

2. F. Celik, M. Guner, "Energy saving device of stator for marine propellers," Ocean Engineering, vol. 34, pp. 850-855, 2007

3. J.P. Chen, J. Su, J.W. Jie, L.Yang, "Investigation on propulsion and flow field of ships with energy saving devices using CFD predictions and model tests," in 12th International Conference on Hydrodynamics, Egmond aan Zee, The Netherlands, September 2016.

4. M. Cheng, C. Hao-peng, Q. Zheng-fang, C. Ke, “The design of propeller and propeller boss cap fins (PBCF) by an integrative method," Journal of Hydrodynamics, vol. 26 (4), pp. 586-593, 2014. 
5. B. W. Coney, A method for the design of a class of optimum marine propulsors. Massachusetts Institute of Technology, 1989.

6. L. Guangnian, Q. Chen, Y. Liu, "Experimental study of dynamic structure of propeller tip vortex," Polish Maritime Research, vol. 27 (2), pp. 11-18, 2020.

7. P. M. Hooijmans, J. Holtrop, J. Windt, "Refitting to save fuel and new approaches in the design of new buildings," in 11th International Symposium on Practical Design of Ships and Other Floating Structures, 2010.

8. L. X. Hou, C. H. Wang, A. K. Hu, F. L. Han, "Wake-adapted design of fixed guide vane type energy saving device for marine propeller," Ocean Engineering, vol. 110(B), pp 11-17, 2015

9. S. Hyun-Joon, L. Jong-Seung, L. Jang-Hoon, MyungRyun Han, H. Eui-Beom, S. Sung-Chul, "Numerical and experimental investigation of conventional and un-conventional preswirl duct for VLCC," International Journal of Naval Architecture and Ocean Engineering, vol. 5(3), pp 414-430, 2013

10. L. Joon-Hyoung, K. Moon-Chan, S. Young-Jin, K. Jin-Gu, "Study on performance of combined energy saving devices for container ship by experiments", Fifth International Symposium on Marine Propulsors smp'17, 2017.

11. J. E. Kerwin, W. B. Coney, C.-Y. Hsin, "Hydrodynamic aspects of propeller/stator design," The Society of Naval Architects and Marine Engineers, Propeller'88 Symposium 5th, 1988.

12. J.-H. Kim, J.-E. Choi, B.-J. Choi, S.-H. Chung, “Twisted rudder for reducing fuel-oil consumption," Ocean Engeering, vol. 6, pp. 715-722, 2014.

13. J.-H. Kim, J.-E. Choi, B.-J. Choi, S-H. Chung, H.-W. Seo, "Development of energy-saving devices for a full slowspeed ship through improving propulsion performance," International Journal of Naval Architecture and Ocean Engineering, vol. 7, pp. 390-398, 2015.

14. M. C. Kim, H. H. Chun, Y. D. Kang, "Design and experimental study on a new concept of preswirl stator as an efficient energy-saving device for slow speed full body ship," SNAME vol 112, pp 111-121, 2004.

15. V. Kraslinikov, K. Koushan, M. Nataletti, L. Sileo, S. Spence, "Design and numerical and experimental investigation of pre-swirl stators PSS," in Sixth International Symposium on Marine Propulsors SMP'19, 2019.

16. P. Król, "Vortex model of ideal guide vane and its application to the real guide vane," in Sixth International Symposium on Marine Propulsors SMP'19, 2019.
17. P. Król, "A new design method of propulsor systems with optimum distribution of bound circulation," Gdańsk University of Technology, 2019.

18. P. Król, T. Bugalski, "Application of vortex flow model in propeller-stator system design and analysis," Polish Maritime Research, vol. 25(1), pp. 24-32, 2018.

19. P. Król, T. Bugalski, M. Wawrzusiszyn, "Development of numerical methods for marine propeller-pre-swirl stator system design and analysis," in Fifth International Symposium on Marine Propulsors smp'17, 2017.

20. P. Król, K. Tesch, "Pre-swirl energy saving device in marine application," in XXIII Krajowa Konferencja Mechaniki Płynów, Zawiercie, 2018.

21. L. Kwi-Joo, A. Jung-Sun, K. Han-Joung, "Comparative study between results of theoretical calculation and model test for performance confirmation of 'Crown Duct,' Journal of Ocean Engineering and Technology, vol. 28(1), pp. 1-5, 2014.

22. S.-S. Lim, T.-W. Kim, D.-M. Lee, C.-G. Kang, S.-Y. Kim, "Parametric study of propeller boss cap fins for container ships," International Journal of Naval Architecture and Ocean Engineering, vol. 6(2), pp. 187-205, 2014; published online Epub6.

23. A. Minchev, M. Schmidt, S. Schnack, "Contemporary bulk carrier design to meet IMO EEDI requirements," Third International Symposium on Marine Propulsors smp'13, 2013.

24. K. Moon-Chan, S. Yong-Jin, L. Won-Joon, L. Joon-Hyoung, "Study on extrapolation method for self-propulsion test with pre-swirl device," Fifth International Symposium on Marine Propulsors smp'17, 2017.

25. A. Nadery, H. Ghassemi, "Numerical investigation of the hydrodynamic performance of the propeller behind the ship with and without WED," Polish Maritime Research, vol. 27(4), pp. 50-59, 2020.

26. H. Nouroozi, H. Zeraatgar, "Propeller hydrodynamic characteristics in oblique flow by unsteady RANSE solver," Polish Maritime Research, vol. 27(1), pp. 6-17, 2020.

27. J. R. Nielsen, W. Jin, "Pre-swirl fins adapted to different operation conditions," Sixth International Symposium on Marine Propulsors SMP'19, 2019.

28. S. Park, G. Oh, S. Rhee, B.-Y. Koo, H. Lee, "Full scale wake prediction of an energy saving device by using computational fluid dynamics," Ocean Engineering, vol. 101, pp. 254-263, 2015.

29. C. D. Simonsen, C. Nielsen, C. Klimt-Møllenbach, C. R. Holm, A. Minchev, "CFD based investigation of potential 
power saving for different rudder types, positions and preswirl fins," Siemens report, November 2012.

30. T. T. Ngoc, D. D. Luu, T. T. H. Nguyen, T. T. T. Nguyen, M. V. Nguyen, "Numerical prediction of propeller - hull interaction characteristics using RANS method," Polish Maritime Research, vol. 26(2), pp. 163-172, 2019.

31. Y. Xing-Kaeding, S. Gatchell, H. Streckwall, “Towards practical design optimization of pre-swirl device and its life cycle assessment," in Fourth International Symposium on Marine Propulsors SMP'15, 2015.

32. S. Yong-Jin, K. Moon-Chan, L. Won-Joon, L. Kyoung-Wan, L. Joon-Hyoung,"Numerical and experimental investigation of performance of the asymmetric pre-swirl stator for container ship," in Fourth International Symposium on Marine Propulsors SMP'15, 2015.

33. J. Zou, G. Tan, H. Sun, J. Xu, Y. Hou, "Numerical simulation of the ducted propeller and application to a semi-submerged vehicle," Polish Maritime Research, vol. 27(2), pp. 19-29, 2020.

34. Y. Zhang, X. Wu, M. Lai, G. Zhou, J. Zhang, "Feasibility study of RANS in predicting propeller cavitation in behind-hull conditions," Polish Maritime Research, vol. 27(4), pp. 26-35, 2020 .

\section{CONTACT WITH THE AUTHOR}

Przemysław Król

e-mail:przemyslaw.krol@cto.gda.pl

Ship Design and Research Centre Szczecińska 65, 80-392 Gdańsk

Poland 